\title{
Adjustable Liners and Sockets for Prosthetic Devices
}

\section{Lenore Rasmussen $^{1 *}$, Simone Rodriguez ${ }^{1}$, Matthew Bowers ${ }^{1}$, Damaris Smith ${ }^{1}$, Greig Martino ${ }^{2}$, Livia Rizzo ${ }^{3}$, Cole Scheiber ${ }^{4}$, Jesse d'Almeida ${ }^{5}$, Curran Dillis ${ }^{6}$}

\author{
${ }^{1}$ Ras Labs, Synthetic Muscle ${ }^{\mathrm{TM}}$ for Prosthetics \& Robotics, USA. \\ ${ }^{2}$ United Prosthetics (UPI), Boston, Massachusetts, USA. \\ ${ }^{3}$ Harvard Medical School, MEDscience Program, USA. \\ ${ }^{4}$ Biology, Tufts University, Massachusetts, USA. \\ ${ }^{5}$ Robotics Engineering, Worcester Polytechnic Institute, Massachusetts, USA. \\ ${ }^{6}$ Hingham HS, Robotics Captain, USA. \\ *Email: rasmuss1@raslabs.com
}

DOI: https://doi.org/10.33137/cpoj.v1i2.32048

\section{INTRODUCTION}

Ras Labs' Synthetic Muscle ${ }^{\mathrm{TM}}$ will allow amputees to continue their active lives without needing to adjust the fitting of their prosthetic device(s) throughout the day. This technology promises to resolve major issues facing amputees, most notably the pain of prosthetic slippage and tissue breakdown. Synthetic Muscle ${ }^{\mathrm{TM}}$, comprising electroactive polymers (EAPs), actively expand or contract at low voltages, while offering impact resistance and pressure sensing, all in one integrated solution. The main objectives of this project is to determine the feasibility of the EAP pads incorporated into prosthetic liners or sockets and to create prototypes of these EAP based shape-morphing pad systems. In collaboration with UPI, testing of these EAP based pads located in strategic areas of the socket was initiated with customers (BK and $\mathrm{AK}$ ) for evaluation and feedback. Characterization of Synthetic Muscle $^{\mathrm{TM}}$ as dual use pressure sensors was initiated. This is a continuation Ras Labs' dynamic prosthetic pad project, which demonstrated how the volume of the EAP can be changed from applying a low voltage and operating temperatures for use in adjustable prosthetic liners and sockets ${ }^{1-8}$.

\section{METHODS}

The EAP networks were developed by polymerization of ionic monomers with specialized cross-linking agents. The desired pore sizes and elasticity in the final networks were achieved by controlling the cross-link density and aqueous solvation during polymerization, followed by equilibration of the EAP in electrolyte solution to its final volume. Pad designs were assembled and tested over contraction and expansion cycles at $1.5 \mathrm{~V}$. A pre-step of expansion for 1 hour (not shown in figures) was performed followed by contraction for 4 hours under load, an expansion cycle for 30 minutes under static load, and an expansion for 5+ hours under $1 \mathrm{~Hz}$ cyclic on-off pressure load to mimic ambulation in the socket. The electroactivity of these pad designs were tested by attaching the reservoir and EAP electrodes to the corresponding polarity for 2-hour contraction and expansion cycles over $1.5 \mathrm{~V}, 3 \mathrm{~V}, 6 \mathrm{~V}$, and $9 \mathrm{~V}$. The dimensions were measured after each cycle to follow the change in height.

\section{RESULTS}

All of the EAP based pad design prototypes performed at $1.5 \mathrm{~V}$, with the $3 \mathrm{lb}$. testing set-up having a higher pound per square inch than the $5 \mathrm{lb}$. testing set-up due to the different sizes of the EAP pads. Contraction occurred over the first 5 hours, static load, followed by 30 minutes expansion, static load, followed by $5+$ hours expansion under cyclic load at $1 \mathrm{~Hz}$ to mimic ambulation. The cyclic load is to demonstrate actuation during daytime use (after a long contraction cycle). In use, these EAP based pads will be contracted overnight at low voltage, then either allowed to expand back to their neutral state at $0 \mathrm{~V}$ or the electric input reversed to provide for expansion. Particularly under cyclic load, expansion is readily observable even within the first cycle, which would be needed for the EAP pads to quickly meet and match void changes from shrinkage of the residual limb if there is a fast change. From the testing at $1.5 \mathrm{~V}, 3 \mathrm{~V}$, $6 \mathrm{~V}$, and $9 \mathrm{~V}$ with 2-hour cycles, all voltage levels showed electroactive actuation. The $3 \mathrm{~V}, 6 \mathrm{~V}$ and $9 \mathrm{~V}$ testing typically demonstrating incrementally more shape-morphing in both expansion and contraction; however, the $9 \mathrm{~V}$ testing produced some artifacts as well with distinct localized effects. As the development of sensing in the EAP progressed, the construction methods improved. A challenge was consistency between the pads themselves, resulting in data that could not be regarded as overly reliable. The solution was to create 
a specific system of manufacturing that was as close as possible for each pad, including: 3D printed molds for the silicone casings and 3D printed pads for a point electrode. Extensive design experiments were performed to select the most suitable system for integration of electrodes within the EAP pads (Figure 1). The most feasible design system is shown, with one electrode as carbon mesh (1x1") and the other as a Pt wire as the point electrode. A silicone mold was poured such that the EAP and electrodes fit in perfectly, with minimal excess casing.

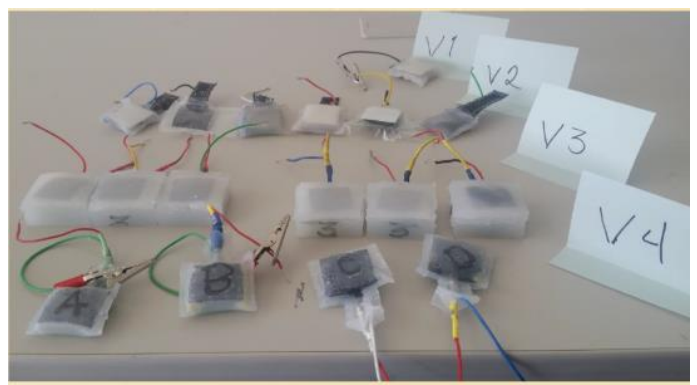

Figure 1. Different versions of EAP based sensors.

\section{Compression Sensing Characterization}

When mechanical pressure is applied to Ras Labs' electroactive polymers, the change in resistance can be recorded. The EAP resistance varies with applied pressure. This has been measured with several different variations of EAPs and encapsulations, with each iteration becoming more consistent and reliable. The EAPs, on average, have a relatively high resistance that varies slightly with pressure. The resistance was measured using an oscilloscope and reading the Wheatstone bridge was attached to EAP (Figure 2). The circuit was attached to a $1 \mathrm{~V}$ amplitude AC signal and the RMS values recorded for each of a series of weight. This allows Ras Labs to use these polymers as resistive sensors in addition to their contractile properties for multiple applications. The sensing capabilities of these EAPs are demonstrated using an oscilloscope and an analog multimeter (Figure 3).

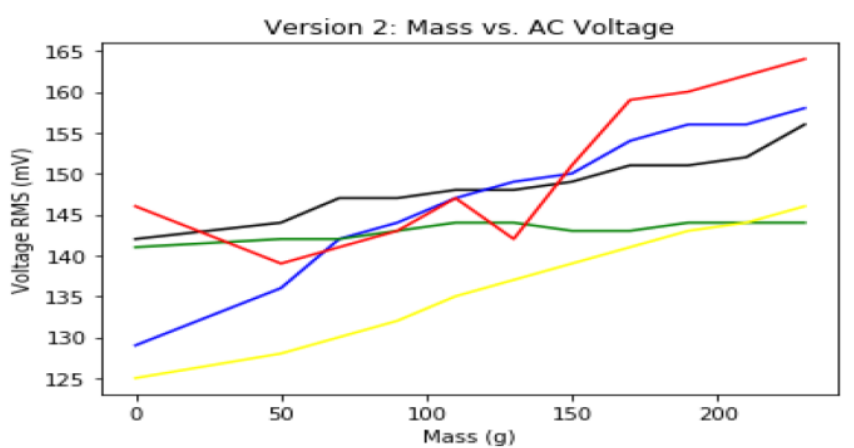

Figure 2. Voltage RMS vs Mass for each of the Version 2 EAPs.

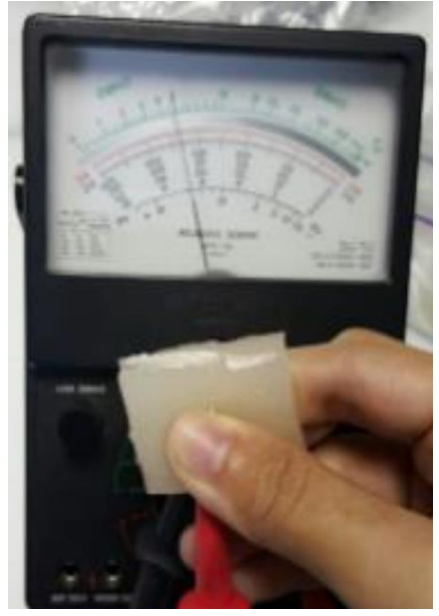

Figure 3. Simple Sensing Demo using Analog Multimeter.

\section{Flexy-Hand 2 Project}

The original files, from Thingiverse, represented Steve Wood and Jeff Erenstone's approach to making a hand for an UL amputee. We started with these open source files, edited them using computer-aided design (CAD) software, and scaled down to the size of our petite patient' hand, working with our customer's anatomy of the vestige hand. A 3D scanner was used to get the patient's mold scanned into the software, which was used to cut a better fit for the 'socket' of the partial hand (Figure 4). By implementing the scan into the established Flexy-Hand 2, the goal was for a more comfortable and more stable fit for our patient during normal usage.
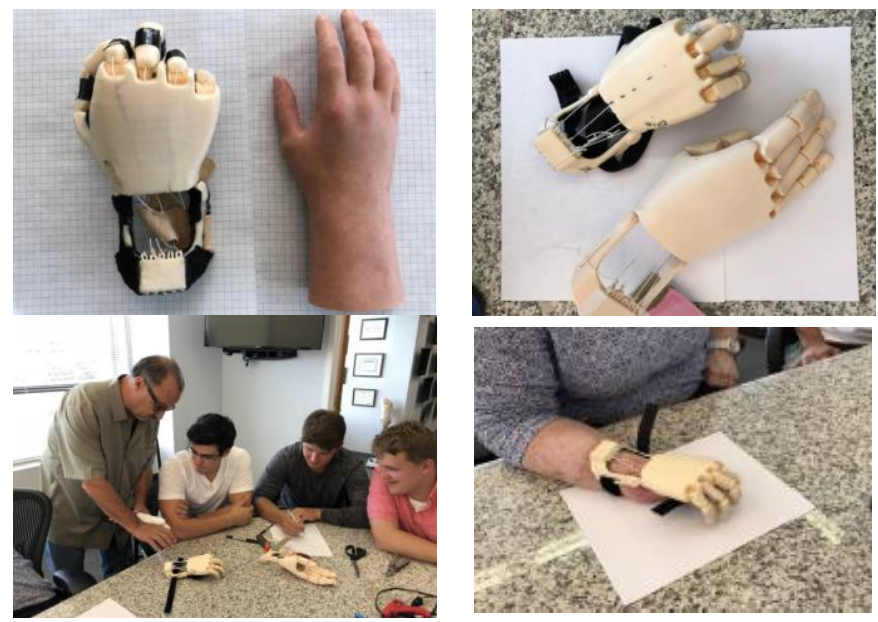

Figure 4. Top Left: The Flexy-Hand 2 (left) next to the modified Flexy-Hand 2 (right): Top Right: The modified Flexy- Hand 2 (bottom) next to the ideal sized hand for this patient (top); Bottom Left: The UPI-Ras Labs' collaborative team constructing the modified hand; Bottom right: The patient wearing the modified Flexy Hand 2. 


\section{CONCLUSIONS AND FUTURE WORK}

The modulation of the EAP shape-morphing control as needed by controlling the voltage level were very promising. This technology is expected to provide for an adjustable prosthetic liner or socket that can maintain dynamic perfect fit throughout the day, for an advanced standard of care for amputees. Ras Labs is working with its collaborators (UPI) and partners to gather evaluation and feedback from $\mathrm{AK}$ and $\mathrm{BK}$ amputees, with promising results. More patient testing is required over longer durations of use. Ras Labs' Synthetic Muscle ${ }^{\mathrm{TM}}$ has the potential to sense pressure and provide for selfadjusting prosthetic liners, with no need for the patient to adjust the fit of their device. As sensors, variable voltage was observed when the EAP sensor was mechanically compressed. Moving towards pixilation of the sensing data will provide a wider range of data and feedback control. The goal is to give amputees natural locomotion with a worry-free prosthesis, maintaining dynamic perfect fit throughout the day and preventing tissue damage from even beginning to occur. With the Flexy- Hand 2 modifications, we are advancing the design to fit any patient's individual needs. Next, we plan to add EAP fingertip sensors to replicate touch while providing life-like compliancy and grip.

\section{ACKNOWLEDGE}

We gratefully acknowledge the National Science Foundation, Breakout Labs, Children's Hospital of Philadelphia/Philadelphia Pediatric Medical Device Consortium, CASIS, US DOE, and US DOD for funding of the synthetic muscle projects. Prosthetic applications are in collaboration with United Prosthetics and additional partners. The MLSC Internship Challenge graciously provided intern support.

\section{REFERENCES}

1.Rasmussen L et al. Synthetic Muscle electroactive polymer (EAP) based actuation and sensing for prosthetic and robotic applications," Proc. SPIE 10594, Electroactive Polymer Actuators and Devices (EAPAD) XX, 105942C. doi: 10.1117/12.2297660, 2018

2.Rasmussen L, Editor, "Electroactivity in Polymeric Materials," Springer-Verlag, GmbH \& Co. KG @ 2012.

3.Rasmussen L, Meixler L.D, Gentile C. Contractile Electroactive Materials and Actuators. SPIE EAPAD 2012, Proc. SPIE 8340, 1O:1-14, 2012.

4.Rasmussen $\mathrm{L}$ et al. Considerations for Contractile Electroactive Polymer Based Materials and Actuators. Proc. SPIE 7976, 2B1-2B13, 2011.
5.Wood S, Erenstone J, Flexy-Hand 2, ttps://www.thingiverse. com/thing:380665 @ 2018

6.Sanders JE, Fatone S. Residual Limb Volume Change: Systematic Review of Measurement and Management. $J$ Rehabil Res Dev, 48(8): 949-986, 2011. DOI:10.1682/JRRD.2010.09.0189

7.Rasmussen L, et al. Considerations for Contractile Electroactive Polymeric Materials and Actuators. Polym. Int. 59: 290-299, 2010. https://doi.org/10.1002/pi.2763

8.Zachariah SG et al. Shape and Volume Change in the Transtibal Residuum over the Short Term. J Rehabil Rev Dev, 41(5): 683-694, 2004. DOI: 10.1682/JRRD.2003.10.0153 